\title{
CUESTIONARIO DE APOYO RECIBIDO DE LA EX PAREJA (CARE): UN INSTRUMENTO BREVE PARA EVALUAR LA CO-PARENTALIDAD POST DIVORCIO
}

\author{
SAGRARIO YÁRNOZ-YABEN \\ Facultad de Psicología, Universidad del País Vasco, San Sebastián
}

\begin{abstract}
Resumen: Presentamos un instrumento para evaluar la percepción de los progenitores divorciados sobre la ayuda que reciben de sus ex parejas para la crianza de los hijos. El CARE (Cuestionario de Ayuda Recibida de la Ex pareja) está compuesto por 8 ítems. Los datos han sido recolectados en cuatro muestras de personas divorciadas y separadas de diversas comunidades del estado y sus hijos. Doscientos veintitrés progenitores y 160 hijos han tomado parte en este estudio. El análisis factorial muestra un factor único, que explica el $41,76 \%$ de la varianza total. El CARE tiene unas adecuadas propiedades psicométricas: buena consistencia interna analizada a través del cálculo del alpha de Cronbach y validez de constructo (convergente y discriminante), demostrada por la relación de esta escala con medidas de adaptación al divorcio (CAD-S) en el caso de los progenitores, y con problemas de conducta (CBCL) en el de los hijos.
\end{abstract}

Palabras clave: Divorcio, adaptación, coparentalidad, problemas de conducta, hijos.

\section{CARE: A short instrument for assessment of post divorce co-parenting.}

\begin{abstract}
This paper presents an instrument for assessment of the perception that divorced parents have about the support they receive from their ex partners in the care for their children. The CARE (Cuestionario de Ayuda Recibida de la Ex pareja) is an 8-item questionnaire created in Spanish. Data were collected in four different samples of divorced and separated persons and their children from different autonomous Spanish communities. Participants were 223 parents and 160 children from divorced families. Factorial analysis came up with a single factor accounting for 41.76 percent of the total variance. The CARE has appropriate psychometric properties: high internal consistency according to Cronbach's alpha and convergent and discriminant construct validity, supported by its relations with measures of adaptation to divorce (CAD-S) in the case of the parents, and with behavioral problems (CBCL) in the case of the children.
\end{abstract}

Keywords: Divorce, adaptation, co-parenting, behavioral problems, children.

\section{INTRODUCCIÓN}

Cuando existen hijos, el divorcio disuelve el matrimonio pero no la familia. Esto hace que

\footnotetext{
Recibido: 29-enero-2010; Aceptado: 7-mayo-2010

Correspondencia: Facultad de Psicología, Departamento PETRA, Avda de Tolosa 70, 20018 San Sebastián. Correo-e: sagrario.yarnoz@ehu.es

Agradecimientos: Este trabajo forma parte de una investigación sobre la adaptación post-divorcio, que ha recibido la ayuda del Vicerrectorado de Investigación de la Universidad del País Vasco-Euskal Herriko Unibertsitatea al grupo de investigación HARREMANAK, código GIU07/61
}

los componentes de la ex pareja deban redefinir sus roles parentales en el contexto cargado de emociones intensas y contrapuestas característico de un divorcio o separación (Yárnoz-Yaben, 2008); estas emociones incluyen hostilidad, afecto, preocupación por la ex pareja, ira, rechazo, pena y ansiedad o pánico.

Diversos estudios resaltan los beneficios que para los hijos tienen la implicación de ambos progenitores en su crianza y el ejercicio de una parentalidad consistente y coordinada. Así, Rodríguez, Del Barrio y Carrasco (2009) encontraron una relación entre la inconsistencia en los hábitos de crianza de los progenitores y la presencia de conductas agresivas y depresivas 
en una muestra de niños y adolescentes con edades comprendidas entre 8 y 17 años. Iglesias y Romero (2009), por su parte, mostraron en pacientes ambulatorios de edades comprendidas entre los 12 y los 19 años, la relación entre la baja aceptación e implicación parental y depresión, y entre el estilo parental autoritario y las alteraciones externalizantes. La situación post divorcio no supone una excepción. Gasper, Stolberg, Macie y Williams (2008) analizaron el papel mediador de la coparentalidad y las prácticas parentales en la asociación entre el estatus marital de los progenitores (divorciados o no) y diversas medidas de ajuste - salud mental general, miedo a la intimidad, autoestima, delincuencia - en estudiantes universitarios.

Una relación de coparentalidad puede ser definida como aquella en la que los dos progenitores interaccionan positivamente, cooperan entre sí y tienen una relación de apoyo mutuo centrada fundamentalmente en la crianza de los hijos, estando ambos implicados activamente en las vidas de sus hijos (Ahrons, 1981). La implicación de ambos progenitores, tome la forma que sea, en las vidas de sus hijos tiene indudables ventajas para éstos, pero también para los progenitores. Por ejemplo, Arditti y Madden-Derdich (1997) encontraron que las madres que tenían custodia compartida con sus ex parejas disfrutaban de relaciones más cooperativas y se sentían mas apoyadas en su rol de madres; los padres, por su parte, se comportaban de una forma más positiva hacia sus ex esposas. Madden-Derdich y Arditti (1999) encontraron también una mejor calidad coparental en los progenitores que tenían arreglos de custodia compartida que en aquellos de custodia única, materna o paterna.

Desgraciadamente, el conflicto parental (McIntosh, 2003; Turner y Kopiec, 2006) y la no adaptación al divorcio de uno o ambos progenitores dificultan, o imposibilitan, la posibilidad de ejercer la coparentalidad. Aunque autores como Braver, Griffin y Cookston (2005) encontraron que la mayoría de los progenitores divorciados experimentan niveles altos de conflicto durante los primeros tres años y después ejercen la parentalidad en paralelo o de forma cooperativa, un $25 \%$ de los progenitores continúa manteniendo el conflicto.
La percepción de que la ex pareja ayuda en la crianza de los hijos está, al parecer, relacionada con la adaptación al divorcio del progenitor que responde, y muy especialmente, con su propia disposición a la coparentalidad (YárnozYaben, 2010). Esta postura de sana colaboración sería la antítesis de cualquier tipo de interferencia parental, entendida como materialización del conflicto post divorcio entre los progenitores, que no solo es dañina para los hijos (McIntosh, 2003; Turner y Kopiec, 2006), sino que también incide negativamente en el bienestar psicológico de los progenitores implicados (Mitcham-Smith y Henry, 2007). También sería lo opuesto del fenómeno conocido como gatekeeping o filtro materno (Allen y Hawkins, 1999), definido como un conjunto de conductas y creencias que inhiben la colaboración entre madres y padres, limitando las posibilidades de los hombres de ocuparse de sus hijos (Cannon, Schoppe-Sullivan, Mangelsdorf, Brown y Sokolowski, 2008).

En nuestra opinión, de cara al desarrollo de estrategias de prevención o intervención para promover una mejor adaptación a su situación de los integrantes de familias divorciadas, algo que tímidamente se comienza a hacer en nuestro medio cultural (p.ej., Yárnoz, Plazaola, y Etxeberria, 2008) es necesario contar con instrumentos que nos permitan evaluar distintos aspectos de dicha adaptación, y el ejercicio de la coparentalidad post divorcio es uno de ellos. El instrumento que aquí presentamos, el Cuestionario de Apoyo Recibido de la Expareja (CARE) tiene como objetivo evaluar de una forma sencilla la percepción del apoyo que progenitores divorciados - padres o madres, custodios o no custodios reciben de su ex pareja, el padre o madre de los hijos habidos en común.

\section{MÉTODO}

\section{Participantes}

En este estudio han participado 223 progenitores divorciados y 160 hijos procedentes de diferentes puntos de España. Estos progenitores eran usuarios-as de Puntos de Encuentro Familiares, de Centros de Apoyo a la Familia del Ayuntamiento de Madrid, padres y madres per- 
tenecientes a asociaciones de divorciados y separados, y finalmente, progenitores divorciados no asociados. La participación fue voluntaria y el único criterio de inclusión que se contempló fue el hecho de estar divorciados o separados y tener hijos-as de esta pareja. La edad media de los progenitores era de 41,21 años $(D T=7,96)$, habían sido pareja durante una media de 11,05 años y llevaban divorciados entre 1 mes y 24 años. El 58\% eran hombres y el $42 \%$ mujeres; un 59\% tenía la custodia de sus hijos, y el $41 \%$ no la tenía. En este estudio, utilizamos además datos de los hijos de estas parejas con una edad comprendida entre $4 \mathrm{y}$ 18 años, edad correspondiente a los criterios de utilización del CBCL (Child Behaviour Checklist, Achenbach 1991). El 58\% eran chicos y el $42 \%$ chicas, y su media de edad era de 5,71 años $(D T=3,80)$.

\section{Elaboración del CARE y procedimiento}

Para la elaboración del cuestionario sobre la percepción de la apoyo recibido de la ex pareja CARE se utilizó inicialmente una base compuesta por 20 ítems, basados en diversos aspectos considerados relevantes en la literatura sobre las relaciones familiares post-divorcio, y que se refieren a la colaboración entre los progenitores (DeGarmo, Patras y Eap, 2008), el ejercicio de la coparentalidad (Bonach, 2005; Cowan, Cowan, Pruett y Pruett, 2007) y el constructo llamado gatekeeping, o filtro (Allen y Hawkins, 1999), es decir, las funciones facilitadoras o inhibidoras ejercidas por uno o ambos progenitores, que determinan quién tiene acceso al hijo, y la naturaleza de ese acceso. Como formato de respuesta se utilizó una escala Likert con cinco alternativas: "Totalmente en desacuerdo» (1), «En desacuerdo» (2), «Ni de acuerdo ni en desacuerdo» (3), «De acuerdo» (4), y «Totalmente de acuerdo» (5).

El cuestionario fue aplicado a una muestra preliminar de personas divorciadas o separadas para comprobar el funcionamiento de los ítems. Se eliminaron aquellos que resultaron ambiguos, redundantes o con una baja frecuencia de respuesta. Asimismo se realizó un análisis factorial mediante el cual se descartaron los ítems que pesaban de manera similar en todos los factores, o cuyo autovalor fuera menor que 0,30 . Por último se analizó exploratoriamente la fiabilidad del instrumento y se descartaron aquellos ítems que al ser eliminados producían un aumento de la consistencia interna. De esta manera el cuestionario de apoyo de la ex pareja quedó conformado por 8 ítems (Véase el Anexo I).

La Confederación Española de Puntos de Encuentro Familiar nos facilitó el contacto con los profesionales situados en diversos puntos del estado. Los profesionales de los Puntos de Encuentro Familiar de Galicia (Ferrol, Vigo, Santiago de Compostela, Ourense, Pontevedra, A Coruña), Euskadi (San Sebastián, Irún, Portugalete, Bilbao), Valencia, Ceuta y Toledo accedieron a participar. Asimismo, hemos contado con la colaboración de profesionales de los Centros de Apoyo a la Familia del Ayuntamiento de Madrid, que ofrecen servicios básicos como información, orientación y atención psicológica y que desde su apertura desde 2006 se estima que han atendido a unas 8000 personas.

El contacto con las asociaciones de padres y madres separados o divorciados se realizó a través de la Confederación Estatal de Padres y Madres separados, de la cual forman parte, entre otras, la Asociación de Padres de Familia Separados de Madrid (APFS), la Federación Andaluza de Padres y Madres Separados (FASE), la Asociación Gallega de Padres y Madres Separados, y la Federación Vasca de Padres y Madres Separados (KIDETZA). El número de asociados puede cifrarse en torno a 40.000 personas.

En el caso de los puntos de encuentro familiar (PEF) y centros de apoyo a la familia (CAF), los trabajadores de los centros explicaron a los usuarios la finalidad de esta investigación y les propusieron que colaboraran en ella. Las personas que accedieron a ello, rellenaron el cuestionario en el centro correspondiente con la ayuda de miembros de su personal, si necesario. En el caso de las asociaciones de padres y madres separados o divorciados, se envió un correo electrónico a la Confederación Estatal de Padres y Madres separados explicando la investigación a realizar y solicitando la colaboración de sus asociados. Posteriormente, se contactó individualmente con aquellas personas 
que se mostraron interesadas en la investigación, a los que se envió por correo (postal o electrónico) los cuestionarios para que los respondieran y una dirección y teléfono de contacto por si tuvieran algún tipo de duda.

\section{Instrumentos de evaluación}

Cuestionario de Adaptación al DivorcioSeparación (CAD-S; Yárnoz-Yaben y CominoGonzález, 2010). Se aplicó para evaluar la adaptación al divorcio. Este cuestionario evalúa aspectos esenciales que reflejan el nivel de adaptación del grupo familiar a la situación de divorcio. El coeficiente a para la escala total es de 0,77. Consta de cuatro factores: (1) Dificultades psicológicas en la adaptación al divorcio-separación $(\alpha=0,78)$, que describe enfado hacia la ex pareja e ideas de que nunca se va a poder superar la separación. (2) Conflicto entre la ex pareja $(\alpha=0,81)$, que refleja discusiones, peleas y desacuerdos con la ex pareja. (3) Disposición a la coparentalidad $(\alpha=0,72)$, que describe una posición favorable a colaborar con la ex pareja para trabajar conjuntamente en beneficio de los hijos. (4) Consecuencias negativas de la separación para los hijos $(\alpha=0,65)$, donde se refleja la creencia del progenitor que responde de que el divorcio ha afectado negativamente a sus hijos: notas más bajas en la escuela, etc. Es posible, asimismo, obtener un índice global de adaptación al divorcio/separación basado en una media ponderada de cada una de las dimensiones, positivas o negativas, que evalúa este instrumento. En esta muestra, la media de adaptación al divorcio era 0,01 y la $D T 1,12$.

Child Behaviour CheckList (CBCL; Achenbach 1991). Se aplicó para evaluar los problemas de conducta de los hijos. El CBCL es un formulario estandarizado para registrar a través de la información obtenida de los padres o madres, los problemas de comportamiento en niños de 4 a 18 años por medio de 113 ítems. Se responde a cada ítem según una escala de 3 puntos, según el grado en que refleje el comportamiento del niño. Sumando los ítems correspondientes, se obtienen las escalas internalizante, externalizante y total. Esta escala posee adecuados índices de fiabilidad y validez refle- jados en un sinnúmero de trabajos (Achenbach, 1985; Cohen, Costello y Mc Consughy, 1982).

Cuestionario de Apoyo Recibido de la Ex pareja (CARE; Anexo I). El Apoyo recibido de la ex pareja se evaluó con el instrumento que aquí presentamos (CARE), compuesto por 8 ítems a los que se responde según una escala de 5 puntos ( 1 totalmente en desacuerdo, 5 totalmente de acuerdo). Las preguntas reflejan la percepción por parte del progenitor que responde (custodio o no custodio) del apoyo que le brinda su ex pareja en la crianza de los niños (en general siento que mi ex pareja entiende y apoya mis necesidades como progenitor, custodio o no) y la satisfacción de los hijos (mis hijos están satisfechos con los arreglos de custodia; p.ej., pueden ver o pueden hablar con el otro progenitor tanto como quieran).

\section{RESULTADOS}

\section{Análisis descriptivos}

La media obtenida en el cuestionario CARE se indica en la Tabla 1. Cuando dividimos la muestra en función del sexo del progenitor (mujeres: media $=19,15 ; D T=6,85$; hombres: media 20,$24 ; D T=6,01$ ) o de la situación de custodia (custodios: media 19,41; DT $=6,87$; no custodios, media 19,$89 ; D T=6,02$ ) las pequeñas diferencias obtenidas resultaron ser carentes de significación estadística (respecto al sexo del progenitor $t=1,23$, n.s.; con respecto a la custodia, $t<1$ ). Realizamos por tanto análisis conjuntos para el total de la muestra. Las medias y desviaciones típicas relativas a las restantes variables pueden observarse en la Tabla 1.

\section{Estructura factorial del Cuestionario de Apoyo Recibido de la Ex pareja (CARE)}

En primer lugar, a fin de comprobar la adecuación muestral para un análisis factorial, se calcularon el índice de Kaiser-Meyer-Olkin (KMO) y el test de esfericidad de Bartlett. El índice KMO mostró un valor de 0,83 y el test de Bartlett resultó estadísticamente significati- 
vo, $\chi^{2}(28)=422,75, p<0,0001$. Así pues, realizamos un análisis de componentes principales con rotación varimax. Siguiendo el criterio de extracción de factores de Kaiser, obtuvimos un único factor con valores propios iguales o superiores a la unidad. Este factor, cuyo autovalor era de 3,34 , explicaba el $41,76 \%$ de la varianza total.

\section{Fiabilidad del Cuestionario de Apoyo Recibido de la Ex pareja (CARE)}

Como medida de fiabilidad se evaluó la consistencia interna del cuestionario mediante el índice $\alpha$ de Cronbach. Obtuvimos un coeficiente $\alpha$ de 0,79 . En la Tabla 2 pueden observarse las saturaciones y las correlaciones total-elemento

Tabla 1. Medias y desviaciones típicas de las variables analizadas

\begin{tabular}{lccc}
\hline Variables & Rango & Media & DT \\
\hline CARE & $8-50$ & 19,60 & 6,52 \\
CAD-S & $-1-+1$ & 0,01 & 1,12 \\
Dificultades & $1-4,67$ & 2,06 & 0,86 \\
Conflicto & $1-5$ & 2,43 & 1,19 \\
Efectos Negat. Hijos & $1-4,67$ & 2,59 & 0,79 \\
Coparentalidad & $1-4,60$ & 2,37 & 0,87 \\
CAD-S total (índice global de adaptación al divorcio) & $-1-+1$ & 0,01 & 1,12 \\
CBCL & & & \\
Internalizante & $0-32$ & 8,24 & 6,81 \\
Externalizante & $0-40$ & 10,09 & 7,53 \\
CBCL Total & $0-118$ & 29,83 & 20,07 \\
\hline
\end{tabular}

Nota: CARE, Cuestionario de Apoyo recibido de la Expareja; CAD-S, Cuestionario de Adaptación al Divorcio-Separación; CBCL, Children Behaviour Checklist.

Tabla 2. Saturaciones y correlación total-elemento de los ítems de la escala CARE

\begin{tabular}{|c|c|c|}
\hline Item CARE & Saturación & $\begin{array}{l}\text { Correlación item- } \\
\text { total corregida }\end{array}$ \\
\hline $\begin{array}{l}\text { 1. En general siento que mi ex pareja entiende y apoya mis necesidades } \\
\text { como progenitor (custodio o no) }\end{array}$ & 0,58 & 0,44 \\
\hline $\begin{array}{l}\text { 2. Cuando yo he necesitado hacer un cambio en los acuerdos de visita, mi } \\
\text { ex pareja ha cedido para que pudiera adaptarme mejor }\end{array}$ & 0,57 & 0,44 \\
\hline $\begin{array}{l}\text { 3. Desde el divorcio mi ex esposo/a ha cumplido con su parte en cuanto a } \\
\text { la crianza y el mantenimiento económico de los niños }\end{array}$ & 0,64 & 0,50 \\
\hline $\begin{array}{l}\text { 4. Yo apruebo las actividades que hacen y el cuidado que reciben mis hi- } \\
\text { jos cuando están con mi ex esposo/a }\end{array}$ & 0,67 & 0,53 \\
\hline $\begin{array}{l}\text { 5. Mis hijos están satisfechos con los arreglos de custodia (p.ej., pueden } \\
\text { ver o pueden hablar con el otro progenitor tanto como quieran) }\end{array}$ & 0,61 & 0,47 \\
\hline $\begin{array}{l}\text { 6. Desde la separación, mi ex pareja y yo estamos de acuerdo sobre la ma- } \\
\text { yoría de las decisiones domésticas }\end{array}$ & 0,73 & 0,60 \\
\hline 7. Mi ex pareja es una ayuda para mí en la crianza de nuestros hijos & 0,76 & 0,63 \\
\hline $\begin{array}{l}\text { 8. Cuando necesito ayuda con respecto a mis hijos, se la pido a su padre/ } \\
\text { madre }\end{array}$ & 0,55 & 0,41 \\
\hline
\end{tabular}


correspondientes a cada uno de los ítems que componen la escala.

\section{Validez de constructo}

A fin de verificar la validez de constructo del instrumento, se analizó en primer lugar la relación del CARE con medidas de adaptación al divorcio para los progenitores, y de problemas de conducta para los hijos. Las correlaciones encontradas, que se reflejan en la Tabla 3, muestran que el apoyo de la ex pareja en la crianza de los niños correlaciona significativamente con la puntuación total de adaptación al divorcio-separación evaluada con el CAD-S y, dentro de este cuestionario, muestra una elevada correlación con la escala de disposición a la coparentalidad.

En segundo lugar, examinamos la adaptación al divorcio de los progenitores y los problemas de conducta encontrados en sus hijos en función del apoyo recibido de la ex pareja. Algunos estudios parecen indicar que existe una relación entre la colaboración entre los progenitores y los problemas de conducta de los hijos (Buehler y Gerard, 2002; Malone et al., 2004), y que la colaboración entre los progenitores está estrechamente relacionada con su nivel de ajuste a la situación de divorcio (Quinney y Fouts, 2003; Markham, Ganong y Coleman, 2007). Así pues, nuestra hipótesis era que los progenitores mejor adaptados al divorcio se permitirían aceptar, y por tanto percibirían, niveles más altos de apoyo por parte del otro progenitor, y que los niños cuyos padres o madres ejercieran una mayor colaboración tras el divorcio-separación manifestarían menos problemas de conducta.

Con respecto a la adaptación al divorcio de los progenitores, comparamos las puntuaciones de aquellos progenitores que decían recibir apoyo de su ex pareja por encima o por debajo de la media (19). Las pruebas de $t$ resultaron significativas para la adaptación total al divorcio y para disposición a la coparentalidad, como puede verse en la Tabla 4.

Respecto a los problemas de conducta en los niños, comparamos las medias obtenidas en el CBCL por los niños cuyos progenitores informaron que recibían apoyo de su ex pareja por encima o por debajo de una puntuación de 19. Como aparece reflejado en la Tabla 4, los datos obtenidos van en el sentido de las hipótesis.

Tabla 3. Correlaciones entre apoyo de la ex pareja, medidas de adaptación al divorcio y problemas de conducta en los hijos

\begin{tabular}{|c|c|c|c|c|c|c|c|c|}
\hline & 2 & 3 & 4 & 5 & 6 & 7 & 8 & 9 \\
\hline 1. Ayuda ex pareja (CARE) & $0,77 * *$ & $-0,03$ & $-0,05$ & $-0,10$ & $0,94 * *$ & $-0,13$ & $-0,14$ & $-0,14$ \\
\hline 2. CAD-S-Total Adaptación al divorcio & & $-0,43 * *$ & $-0,52 * *$ & $-0,48 * *$ & $0,77 * *$ & $-0,24 * *$ & $-0,22 * *$ & $-0,23 * *$ \\
\hline 3. CAD-S Dificultades & & & $0,34 * *$ & $0,39 * *$ & 0,04 & $0,27 * *$ & $0,25 * *$ & $0,24 * *$ \\
\hline 4. CAD-S Conflicto & & & & $0,36^{* *}$ & 0,00 & 0,09 & 0,09 & 0,07 \\
\hline 5. CAD-S Efectos negativos hijos & & & & & $-0,02$ & $0,27 * *$ & 0,14 & $0,23 * *$ \\
\hline 6. CAD-S Coparentalidad & & & & & & $-0,11$ & $-0,13$ & $-0,12$ \\
\hline 7. CBCL Internalizante & & & & & & & $0,56^{* *}$ & $0,87 * *$ \\
\hline 8. CBCL Externalizante & & & & & & & & $0,84 * *$ \\
\hline 9. CBCL Total & & & & & & & & \\
\hline
\end{tabular}


Tabla 4. Adaptación al divorcio (progenitores e hijos) en función del apoyo recibido de la ex pareja

\begin{tabular}{|c|c|c|c|c|c|}
\hline \multirow[t]{2}{*}{$\begin{array}{l}\text { Variables progenitores } \\
(n=223)\end{array}$} & \multicolumn{2}{|c|}{$\begin{array}{c}\text { Apoyo recibido } \\
\geq 19,00(n=113)\end{array}$} & \multicolumn{2}{|c|}{$\begin{array}{l}\text { Apoyo recibido } \\
<19,00(n=110)\end{array}$} & \multirow[t]{2}{*}{$t$} \\
\hline & Media & $D T$ & Media & $D T$ & \\
\hline CAD-S-Total Adaptación divorcio & 0,74 & 0,93 & $-0,74$ & 0,76 & $13,07 * * *$ \\
\hline CAD-S Dificultades & 2,04 & 0,88 & 2,10 & 0,83 & $-0,54$ \\
\hline CAD-S Conflicto & 2,38 & 1,12 & 2,49 & 1,26 & $-0,67$ \\
\hline CAD-S Efectos negativos hijos & 2,51 & 0,68 & 2,68 & 0,89 & $-1,58$ \\
\hline CAD-S Coparentalidad & 3,05 & 0,61 & 1,68 & 0,45 & $19,04 * * *$ \\
\hline \multirow[t]{2}{*}{ Variables hijos $(n=160)$} & \multicolumn{2}{|c|}{$\begin{array}{c}\text { Apoyo recibido } \\
\geq 19,00(n=75)\end{array}$} & \multicolumn{2}{|c|}{$\begin{array}{l}\text { Apoyo recibido } \\
<19,00(n=85)\end{array}$} & $t$ \\
\hline & Media & $D T$ & Media & $D T$ & \\
\hline CBCL Internalizante & 6,83 & 6,17 & 9,49 & 7,14 & $-2,53 * *$ \\
\hline CBCL Externalizante & 9,36 & 6,84 & 10,74 & 8,07 & $-1,17$ \\
\hline CBCL Total & 26,58 & 19,40 & 32,69 & 20,32 & $-1,94 *$ \\
\hline
\end{tabular}

Nota: CAD-S, Cuestionario de Adaptación al Divorcio-Separación; CBCL, Children Behaviour Checklist. $* p<.05 ; * * p<.01 ; * * * p<.001$.

\section{DISCUSIÓN}

El objetivo del presente estudio ha consistido en elaborar un cuestionario que evalúe el apoyo recibido por parte de la ex pareja en la crianza de los hijos en una situación post-divorcio, y en realizar un análisis de sus características psicométricas.

Los resultados obtenidos parecen poner de manifiesto que el Cuestionario de Apoyo Recibido de la Ex pareja (CARE) posee un adecuado nivel de consistencia interna. En lo que respecta a la validez del instrumento, el análisis de componentes principales llevado a cabo para examinar su dimensionalidad mostró una estructura unifactorial. Además, parece que el CARE posee una adecuada validez de constructo: nuestros datos sugieren que las puntuaciones obtenidas en el CARE correlacionan con las puntuaciones obtenidas por los progenitores en la evaluación de su ajuste al divorcio medida con el CAD-S (puntuación total y Disposición a la coparentalidad), aunque no con medidas de problemas de conducta de los hijos. Niveles de apoyo de la expareja por encima o por debajo de la media (19) se corresponden con una ma- yor, o menor en su caso, adaptación de los progenitores al divorcio, y con menores o mayores problemas de conducta de los hijos.

A partir de los datos obtenidos en el presente estudio, cabe concluir que el CARE cumple con los requisitos metodológicos para evaluar el nivel de colaboración de la ex pareja en la crianza de los hijos tras el divorcio. Varios estudios han demostrado que no es el divorcio en sí lo que compromete diversos aspectos del funcionamiento normal de los niños y los adultos, sino el nivel de conflicto marital y parental (Johnston y Campbell, 1988; McIntosh, 2003; Pons-Salvador y Del Barrio, 1995), y las consecuentes relaciones desestabilizadas de los hijos con los progenitores que éste genera (King y Heard, 1999), que influyen negativamente sobre la dinámica afectiva de los hijos de personas divorciadas. Un riesgo habitual en estas situaciones es que las madres custodias subestimen el valor de la aportación del padre al bienestar de los hijos (Manning y Lamb, 2003) y la importancia de que los hijos mantengan relaciones cercanas y cálidas con ambos progenitores. Por ejemplo, King y Sobolewski (2006) encontraron que, aunque la calidad de las relaciones con la 
madre parece tener más importancia en el bienestar psicológico de los hijos, unas relaciones estrechas con el padre no residente estaban relacionadas con menores puntuaciones en problemas de conducta evaluados con el CBCL.

En definitiva, creemos que el Cuestionario de Apoyo Recibido de la Ex pareja, CARE, evalúa un elemento tan fundamental en la dinámica de la adaptación post-divorcio del grupo familiar como es el ejercicio de la coparentalidad (Yárnoz-Yaben, 2010). Al ser de pasación sencilla, y estar compuesto por un número relativamente reducido de ítems, permite al profesional que trabaja en el ámbito del divorcioseparación obtener fácilmente una evaluación del nivel de apoyo recibido de la ex pareja en la crianza de los hijos tras el divorcio. Este sencillo instrumento puede suponer una ayuda en la toma de decisiones judiciales (Arce, Fariña y Seijo, 2005), así como para evaluar los resultados de programas realizados con el objetivo de fomentar la coparentalidad (Cowan, Cowan, Pruett y Pruett, 2007) o el trabajo realizado en los Puntos de Encuentro o dispositivos similares.

\section{REFERENCIAS}

Achenbach, T. M. (1991). Manual for the Child Behavior Checklist 4/18 y 1991 Profile. Burlington, VT: University of Vermont. Department of Psychiatry.

Achenbach, T. M. (1985). Assessment and taxonomy of child and adolescent psycopathology. Stanford: Sage

Ahrons, C. R. (1981). The continuing coparental relationships between divorced spouses. American Journal of Orthopsychiatry, 51: 415-428.

Allen, S. M. y Hawkins, A. J. (1999). Maternal gatekeeping: mothers' beliefs and behaviors that inhibit greater father involvement in family work. Journal of $\mathrm{Ma}$ rriage and the Family, 61, 199-212.

Arce, R., Fariña, F., y Seijo, D. (2005). Razonamientos judiciales en procesos de separación. Psicothema, 17, 57-63.

Arditti, I. A., y Madden-Derdich, D. (1997). Joint and sole custody mothers: Implications for research and practice. Families in Society, 78, 36-45.

Braver, S. L., Griffin, W. A., y Cookston, J. T. (2005). Prevention programs for divorced nonresident fathers. Family Court Review, 43, 81-96.

Bonach, K. (2005). Factors contributing to quality coparenting: implications for family policy. Journal of Divorce \& Remarriage, 43, 79-103.
Buehler, C., y Gerard, J. M. (2002). Marital conflict, ineffective parenting and children's and adolescent's maladjustment. Journal of Marriage and Family, 64, 78-92.

Cannon, E. A., Schoppe-Sullivan, S. J., Mangelsdorf, S. C., Brown, G. L., y Sokolowski, M. S. (2008). Parent characteristics as antecedents of maternal gatekeeping and fathering behavior. Family Process, 47, 501-519.

Cohen, N. J., Costello, E. I., y Mc Consughy, S. H. (1982). The relationship between children's internalizing and externalizing sympton patterns and mesures of competence: A study of the concurrent validity of the Achenbach Child Behavior Profile. Dallas: American Academy of Child Psychiatry.

Cowan, C. P., Cowan, P. A., Pruett, M. K., y Pruett, K. (2007). An approach to preventing coparenting conflict and divorce in low-income families: strengthening couple relationships and fostering fathers' involvement. Family Process, 46, 109-121.

Cronbach, L. J. (1951). Coefficient alpha and the internal structure of tests. Psychometrika, 16, 297-334.

DeGarmo, D. S., Patras, J., y Eap, S. (2008). Social support for divorced fathers' parenting: testing a stress-buffering model. Family Relations, 57, 35-48.

Gasper, J. A. F., Stolberg, A. L., Macie, K. M. y Williams L.J. (2008). Coparenting in intact and divorced families: its impact on young adult adjustment. Journal of Divorce y Remarriage, 49, 272-290.

Iglesias, B. y Romero, E. (2009). Estilos parentales percibidos, psicopatología y personalidad en la adolescencia. Revista de Psicopatología y Psicología clínica, 14, 63-77.

Johnston, J. R., y Campbell, L. E. (1988). Impasses of divorce: The dynamics and resolution of family conflict. New York: Free Press.

King, V., y Heard, H. E. (1999). Nonresident father visitation, parental conflict, and mother's satisfaction: What's best for child well-being? Journal of Marriage and the Family, 61, 385-396.

King, V., y Sobolewski, J. M. (2006). Nonresident fathers' contributions to adolescent well-being. Journal of Marriage and Family, 68, 537-557.

McIntosh, J. (2003). Enduring conflict in parental separation: pathways of impact on child development. Journal of Family Studies, 9, 63-80.

Madden-Derdich, D. A., y Arditti, J. A. (1999). The ties that bind: Attachment between former spouses. Family Relations, 48, 243-249.

Malone, P. S., Lansford, J. E., Castellino, D. R., Berlin, L. J., Dodge, K. A., Bates, J. E., y Pettit, G. S. (2004). Divorce and child behavior problems: Applying latent change score models to life event data. Structural Equation Modeling: A Multidisciplinary Journal, 11, 401-423.

Manning, W., y Lamb, K. A. (2003). Adolescent wellbeing in cohabiting, married, and single-parent families. Journal of Marriage and the Family, 65, 876-893. 
Markham, M. S., Ganong, L. H., y Coleman, M. (2007). Coparental identity and mothers' cooperation in coparental relationships. Family Relations, 56, 369-377.

McIntosh, J. (2003). Enduring conflict in parental separation: pathways of impact on Child Development. Journal of Family Studies, 9, 63-80.

Mitcham-Smith, M., y Henry, W. J. (2007). High-conflict divorce solutions: parenting coordination as an innovative co-parenting Intervention. The family Journal: counseling and therapy for couples and families, 15, 368-373.

Pons-Salvador, G., y Del Barrio, M. V. (1995). El efecto del divorcio sobre la ansiedad de los hijos. Psicothema, 7, 489-497.

Quinney, D. M., y Fouts, G. T. (2003). Resilience and divorce adjustment in adults participating in divorce recovery workshops. Journal of Divorce \& Remarriage, 40, 35-68.

Rodriguez, M. A., Del Barrio, V. y Carrasco, M. A. (2009). Consistencia interparental y su relación con la agresión y la sintomatología depresiva en niños y adolescentes.
Revista de Psicopatología y Psicología Clínica, 14, 51-60.

Turner, H. A., y Kopiec, K. (2006). Exposure to interparental conflict and psychological disorder among young adults. Journal of Family Issues, 27, 131-158.

Yárnoz-Yaben, S. (2008). El divorcio como un proceso de pérdida y duelo. Aportaciones de la Teoría del Apego. En S. Yárnoz Yaben (comp.), La teoría del apego en la clínica, pp. 187-212. Madrid: Psimática.

Yárnoz-Yaben, S. (2010). Hacia la coparentalidad postdivorcio: percepción del apoyo recibido de la ex pareja en progenitores divorciados españoles. International Journal of Health Psychology 10, 295-307.

Yárnoz-Yaben, S. y Comino-González, P. (2010). El CAD-S, un cuestionario para la evaluación de la adaptación al divorcio-separación. Psicothema 22, 157-162.

Yárnoz, S., Plazaola, M., y Etxeberria, J. (2008). Adaptation to divorce: An Attachment-based intervention with long-term divorced parents. Journal of Divorce \& Remarriage, 49, 291-307. 


\section{ANEXO I \\ Cuestionario DE APOYO RECIBIDO DE LA Ex PAREJA (CARE)}

1. En general siento que mi ex pareja entiende y apoya mis necesidades como progenitor (custodio o no).

2. Cuando yo he necesitado hacer un cambio en los acuerdos de visita, mi ex pareja ha cedido para que pudiera adaptarme mejor.

3. Desde el divorcio mi ex esposo/a ha cumplido con su parte en cuanto a la crianza y el mantenimiento económico de los niños.

4. Yo apruebo las actividades que hacen y el cuidado que reciben mis hijos cuando están con mi ex esposo/a.

5. Mis hijos están satisfechos con los arreglos de custodia (p.ej., pueden ver o pueden hablar con el otro progenitor tanto como quieran).

6. Desde la separación, mi ex pareja y yo estamos de acuerdo sobre la mayoría de las decisiones domésticas.

7. Mi ex pareja es una ayuda para mí en la crianza de nuestros hijos.

8. Cuando necesito ayuda con respecto a mis hijos, se la pido a su padre/madre.

Se responde según una escala de 5 puntos (1 totalmente en desacuerdo, 5 totalmente de acuerdo).

Normas de corrección: La puntuación total se obtiene sumando el valor de las respuestas dadas a cada uno de los ítems. La puntuación obtenida refleja la percepción por parte del progenitor que responde (custodio o no custodio) del apoyo que le brinda su ex pareja en la crianza de los niños. 